\title{
Ein visueller Metadaten Browser für die explorative Erkundung großer Datenmengen
}

\author{
Harald Reiterer, Tobias Limbach, Frank Müller, Peter Klein, Christian Jetter \\ Universität Konstanz, Fachbereich Informatik \& Informationswissenschaft, Arbeitsgruppe \\ Mensch-Computer Interaktion
}

\section{Zusammenfassung}

\begin{abstract}
Es wird ein visuelles Suchsystem zur explorativen Erkundung großer Datenmengen vorgestellt. Das System VisMeB (Visueller Metadaten-Browser) basiert auf einer Reihe von Designstudien, die im Rahmen unterschiedlicher Forschungsprojekte entwickelt worden sind. Der besondere Schwerpunkt lag dabei auf der Entwicklung innovativer Visualisierungs- und Interaktionstechniken, die den Benutzer von der Anfrageformulierung bis zur Ergebnisdarstellung unterstützen. Die anhand eines szenarienbasierten Usability Engineering Ansatzes entwickelten Designstudien wurden einer Reihe von Evaluationen unterzogen. Daraus ergaben sich zahlreiche Redesignvorschläge, die bereits umgesetzt wurden.
\end{abstract}

\section{Einleitung}

Zentrale Idee des hier beschriebenen Forschungsvorhabens ist die Gestaltung der MenschComputer Interaktion (MCI) im Zusammenhang mit der Exploration und Visualisierung großer Datenbestände, wie man sie beispielsweise in Digitalen Bibliotheken, im Web, in Data Warehouses oder in Produktdatenbanken von E-Commerce-Anwendungen findet. Vom Standpunkt der MCI bestehen die besonderen Herausforderungen darin, durch die Entwicklung bisher nicht verfügbarer interaktiver, visueller Artefakte und neuer Interaktionstechniken ganz neue Formen der visuellen MCI zu ermöglichen. Hier wird das Feld des traditionellen GUI Designs verlassen, das mittlerweile auch forschungsmäßig gut abgesichert ist und nach völlig neuen Wegen der visuellen Interaktion mit dem Medium Computer gesucht.

Der im Rahmen dieses Vorhabens entwickelte Metadatenbrowser ist ein Beispiel für einen derartigen neuen Weg der visuellen Interaktion und soll den Benutzer bei der explorativen Erkundung großer Datenbestände unterstützen. Neben dem freien Erkunden der Inhalte eines Datenbestandes steht hier vor allem die Unterstützung der gezielten Suche nach bestimmten Daten zur Befriedigung eines konkreten Informationsbedürfnisses (z. B. Welche Dokumente bzw. Geodaten sind für die Planung des Standortes eines Supermarktes verfügbar?) im Mittelpunkt der Betrachtung. Dabei gilt es den Benutzer sowohl bei der Formulierung seiner Suchanfrage visuell zu unterstützen als auch beim Herausfiltern der für ihn relevanten Daten aus der oft sehr großen Treffermenge einer Suchanfrage. Eine Zielstellung, die im Zeitalter der Informationsüberflutung - aufgrund der Vielzahl der mittlerweile verfügbaren digitalisierten Datenbestände - immer mehr an Bedeutung gewinnt.

Um diese Zielstellung zu erreichen, haben wir - basierend auf fremden und eigenen Vorarbeiten (siehe Kapitel 2) - im Rahmen eines Forschungsprojektes namens VisMeB einen Visuellen Metadaten-Browser entwickelt, der in unterschiedlichen Anwendungsdomänen (siehe Kapitel 3) zum Einsatz kommen kann. Der Metadatenbrowser wurde als generisches Framework zur Visualisie- 
rung von unterschiedlichen Metadaten konzipiert. Mittels einer eigenen Konfigurationskomponente kann eine konkrete Anpassung der Visualisierungen und der notwendigen Retrieval Verfahren auf die jeweiligen Metadaten der konkreten Anwendungsdomäne vorgenommen werden (z. B. in Abhängigkeit des jeweiligen Datentyps). Die grundlegende Hypothese unseres Forschungsprojektes lautet: Die in VisMeB angebotenen Visualisierungen und Interaktionstechniken ermöglichen einen effektiveren und effizienteren Informationsgewinnungsprozess als traditionelle listen- bzw. tabellenbasierte Suchsysteme.

Ein wesentlicher Schwerpunkt des Vorhabens besteht in der umfassenden Evaluation aller Designstudien mittels Methoden des Usability Engineering (Benutzertests im Usability Lab, online Fragebögen), um deren Machbarkeit und Gebrauchstauglichkeit (im Sinn der EN ISO 9241 11) zu überprüfen. Einem szenario-basierten Usability Engineering Prozess (vgl. Rosson und Carroll 2002) folgend, wurden bereits eine Reihe von Papier- und digitalen Mockups sowie erste Java Prototypen entwickelt. Diese wurden sowohl hinsichtlich der technischen Machbarkeit (Feasability im Sinne eines „Proof of Concepts“) als auch hinsichtlich der Gebrauchstauglichkeit (Usability) evaluiert (siehe Kapitel 4). Daraus ergaben sich eine Reihe von Redesignvorschlägen, die derzeit umgesetzt werden. Die der Entwicklung von VisMeB zugrunde liegende Hypothese soll in einem abschließenden, komparativen Benutzertest geprüft werden (Vergleich mit einem Kontrollsystem, das nur über einfache listen- bzw. tablellenbasierte Darstellungen verfügen wird) (siehe Kapitel 5).

\section{Vorarbeiten}

Eine Reihe von Mockups und Prototypen wurden im Rahmen von zwei EU Forschungsprojekten zur Unterstützung von Rechercheaufgaben entwickelt und gingen als Vorarbeiten in die wesentlich umfassenderen Forschungsideen des Vorhabens VisMeB ein. Im EU Forschungsprojekt INSYDER $^{1}$ (Information Systeme de Recherche) wurde eine visuelle Suchmaschine für das Web entwickelt (vgl. Reiterer et al. 2000 und Reiterer et al. 2001), die neben der traditionellen Listendarstellung der Suchergebnisse über weitere Visualisierungsformen zur Darstellung der Suchergebnisse verfügte (z. B. Tabelle mit eingebetteter Relevanzkurve, Punktdiagramm, Balkendiagramme, Stapeldiagramme, TileBars).

Die Erfahrungen des Forschungsprojektes INSYDER flossen in ein nachfolgendes EU Forschungsprojekt namens INVISIP ${ }^{2}$ (Information Visualization for Site Planning) ein (vgl. Göbel et al. 2002). Auch hier wird ein visuelles Suchsystem entwickelt, das Planer beim Finden von entscheidungsrelevanten Daten für die Standortplanung unterstützen soll. Die im Rahmen des Forschungsprojektes INSYDER entwickelten Visualisierungen wurden einer umfassenden empirischen Evaluation mit 40 Benutzern unterzogen (vgl. Mann 2002). Die Erkenntnisse dieser Evaluation, mit Schwerpunkt auf der Beurteilung der Gebrauchstauglichkeit dieser Visualisierungen, führten zur Entwicklung neuer Visualisierungen namens SuperTable + Scatterplot (vgl. Klein et al. 2002). Diese neu entwickelten Visualisierungen greifen ein klassisches Thema der Informationsvisualisierung auf: Einerseits soll ein einzelnes Datenelement hinreichend detailliert dargestellt werden, um es interpretierten zu können. Andererseits sollte das jeweilige Datenelement im Kon-

\footnotetext{
${ }^{1}$ EU ESPRIT Project No. 29232 INSYDER

${ }^{2}$ EU IST-2000-29640 INVISIP
} 
text des gesamten Informationsraumes sichtbar bleiben, um Vergleiche mit anderen Datenelementen durchführen zu können. Nur dadurch kann eine Interpretation eines Datenelementes im Kontext ermöglicht werden. Die größte Herausforderung hinsichtlich der Gestaltung der Visualisierungen besteht darin, die konzeptionelle Verknüpfung zwischen der Detailansicht (ein bzw. wenige Datenelemente) und dem Überblick (möglichst alle Datenelemente) in einer intuitiven Art und Weise zu ermöglichen. Dieses grundlegende Problem der Informationsvisualisierung hat viele Lösungsansätze erfahren, wie beispielsweise Fisheye Views, Focus \& Context Techniken, Multiple-Window User Interfaces und viele weitere mehr. Zur Vereinfachung wollen wir all diese Lösungsansätze unter dem Oberbegriff „Overview \& Detail“ Techniken zusammenfassen.

Die SuperTable folgt dem Lösungsansatz der Focus \& Context Techniken (vergleichbar der TableLense von Rao und Card 1994). Nach Möglichkeit werden alle Ergebnisse einer Suchanfrage in kompakter Art und Weise in der SuperTable angezeigt, um einen Gesamtüberblick zu ermöglichen. Um dies zu erreichen, müssen die auf der ersten Darstellungsebene genutzten Visualisierungen eine kompakte, wenige Pixel an Höhe benötigende Darstellung ermöglichen. Dazu werden Balkendiagramme (Bar Graphs) genutzt (siehe Abbildung 2). Beim Überfahren der jeweiligen Zeilen der Tabelle mit der Maus (focus) ermöglicht eine Art Vorschaufunktion das Einblenden der zweiten Darstellungsebene (die mehr Details bietet), wobei aber der Kontext mit den anderen Datenelementen der Tabelle erhalten bleibt (siehe Abbildung 2, eingerahmte Zeile oben). Jede weitere Detaillierungsebene bringt neue Visualisierungen (z. B. detaillierte Bar Graphs, TileBars, Relevance Curve), die eine immer detailliertere Darstellung der Daten ermöglichen, allerdings um den Preis des höheren Platzbedarfes. Diese unterschiedlichen Detaillierungsgrade ermöglichen den Benutzern eine auf das jeweilige Informationsbedürfnis angepasste Informationspräsentation.

Für die SuperTable wurden zwei Design-Varianten entwickelt, die LevelTable, und die GranularityTable, die dieses Granularitätskonzept in unterschiedlicher Art und Weise umsetzen. Bei der LevelTable können die Sucherergebnisse (z. B. Dokumente) in vier verschiedenen Detaillierungsgraden (4 Ebenen) betrachtet werden, wobei ein Wechsel des Detaillierungsgrades alle Suchergebnisse betrifft (siehe Abbildung 5). Die geringste Granularität und damit die wenigsten Details, aber dafür einem Gesamtüberblick, bietet dabei die erste Ebene. Die höchste Granularität, nämlich letztendlich den Originaldatensatz, bietet die vierte Ebene. Die zweite Design-Variante, von uns GranularityTable genannt, ermöglicht die gleichzeitige Betrachtung von Suchergebnissen in unterschiedlichen Detaillierungsgraden (siehe Abbildung 3). Zwischen der ersten Ergebnisvisualisierung (geringe Granularität) und dem letztendlichen Darstellen des Originaldatensatzes (höchste Granularität) gibt es je Datenelement unterschiedliche Detaillierungsgrade - die Visualisierungen wandeln sich ständig und können, wenn von den Benutzern gewünscht, in verschiedenen Schritten dem Originaldatensatz entgegengehen. Dieses Granularitätskonzept, das sich vom Terminus an der Photographie orientiert (hier bezeichnet dieser Terminus die Auflösung bzw. Körnung eines Films), erstreckt sich über sechs Detaillierungsgrade.

Der Scatterplot (Punktdiagramm) ist eine zusätzliche graphische Repräsentation der Ergebnismenge und ermöglicht einen schnellen Überblick über die Treffermenge (siehe Abbildung 2). Er wurde vom INSYDER System übernommen, da einige der Benutzer das Punktdiagramm als Überblicksvisualisierung bevorzugten. Allerdings wurde auch der Scatterplot um eine Focus \& Context Technik (Bifocal Display mit Verzerrung in der x- und y-Achse) erweitert, um ähnlich wie die SuperTable dem Anspruch des Overview \& Detail gerecht zu werden. Des weiteren wurden eine Reihe von neuen Interaktionstechniken eingeführt (z.B. Moveable Filter), auf die im Abschnitt 3 näher eingegangen wird. 
Es gibt mittlerweile eine Fülle von visuellen Suchsystemen für die Suche in unterschiedlichsten Anwendungsdomänen. Einen guten Überblick über den Stand der Forschung gibt die Arbeit von Mann (vgl. Mann 2002). Im folgenden werden nur Systeme erwähnt, welche die hier vorgestellten Arbeiten wesentlich beeinflusst haben. Die Verwendung des Scatterplots (Punktdiagramm) wurde durch Systeme wie den FilmFinder (vgl. Ahlberg und Shneiderman 1994) und Envision (vgl. Nowell et al. 1996) motiviert. Der Einsatz von visuellen Filtern in Kombination mit dem Scatterplot wurde von der Idee der Moveable Filters beeinflusst (vgl. Fishkin und Stone 1995). Die Idee des Bifocal Display in den Scatterplot zu integrieren geht auf (Spence und Apperley 1982) zurück. Der Einsatz von Balkendiagrammen für die Visualisierung von Gesamt- und Teilrelevanzen von Suchergebnissen im Bereich des Dokumenten Retrievals basiert auf Ideen des Systems Inquery (vgl. Verassamy und Navathe 1995). TileBars zur Darstellung der Verteilung der Relevanzwerte der einzelnen Suchbegriffe innerhalb eines Dokumentes wurden von Hearst entwickelt (vgl. Hearst 1995). Die tabellarische Darstellung der Suchergebnisse in Kombination mit Focus + Context Techniken wurde von den Visualisierungen der Systeme Table Lense (vgl. Rao und Card 1994) und FOCUS (vgl. Spenke et al. 1996) beeinflusst. Die Synchronisation der Visualisierungen im SuperTable + Scatterplot mittels Brushing and Linking wurden durch Arbeiten von North (vgl. North und Shneiderman 2000) inspiriert. Die Idee des Granularitätskonzeptes entstand in Diskussionen mit Maximilian Eibl und basiert auf Erkenntnissen, die er bei der Entwicklung des visuellen Information Retrieval Systems DEViD gewonnen hatte (vgl. Eibl 2002). Der Einsatz von Query Previews als visuelle Vorschaufunktion für online Datenbanken greift auf Arbeiten von Tanin zurück (vgl. Tanin et al. 2000).

\section{$3 \quad$ Anwendung und Einsatz}

Abbildung 1 zeigt die grundlegenden Komponenten von VisMeB. Das System ist in einer Javabasierten Client-Server Architektur realisiert, die sowohl als eigenständige Applikation als auch als Applet innerhalb eines Internetbrowsers genutzt werden kann. Auf dem Server werden alle Benutzer-, Konfigurations- und sitzungsbezogenen Daten gespeichert. Damit kann der Benutzer von beliebigen Arbeitsplätzen auf seine spezifischen Einstellungen zurückgreifen. Zur visuellen Unterstützung der Formulierung der Suchanfrage wird ein anwendungsspezifisches Suchformular mit Vorschaufunktion und Präselektion (Query Preview als Circle Segment View, siehe Abbildung 1) verwendet. Der Zugriff kann nun auf verschiedene Metadatenbanken erfolgen (z. B. Webdokumente, Geometadaten, Filmdaten). Die Ergebnisse der Suchanfrage werden sowohl mit Hilfe von tabellenbasierten (SuperTable) als auch punktdiagrammbasierten (Scatterplot) Visualisierungen dargestellt. Beide Visualisierungsformen sind hinsichtlich ihres Interaktionsverhaltens (Look and Feel) synchronisiert.

Einer der Vorteile von VisMeB ist die Flexibilität bezüglich der Anwendungsbereiche. VisMeB ist nicht auf eine spezielle Domäne fixiert, sondern kann dank des flexiblen Datenmodells entsprechend angepasst werden. Um diese Flexibilität zu testen, wurde VisMeB bisher für verschiedene Anwendungsdomänen entwickelt und gestestet. So gibt es derzeit Anbindungen an eine Filmdatenbank, eine Geometadatenbank und, in der Tradition von INSYDER stehend, eine Anbindung ans Web.

Im folgenden Einsatzszenario wird die Filmdatenbank zur Darstellung von VisMeB genutzt. Sie enthält Metadaten von mehreren hundert Filmen, wie z. B. Titel, Jahr, Sprache, Genre, Bewertung sowie eine Kurzbeschreibung. Ein typisches Einsatzszenario ist die Suche nach einem passenden 
Film für eine bestimmte Situation (z. B. eine Komödie nach einem anstrengenden Tag). Der Benutzer formuliert ähnlich wie bei einer Suchmaschine im Web mittels einigen Suchtermen eine Suchanfrage und startet die Suche. Geht man davon aus, dass das letztlich Entscheidende für eine Auswahl der Inhalt, also die Kurzbeschreibung des Films ist, hat der Benutzer jetzt mehrere Möglichkeiten vorzugehen, welche am Beispiel der Kombination GranularityTable/Scatterplot auszugsweise näher erläutert werden.

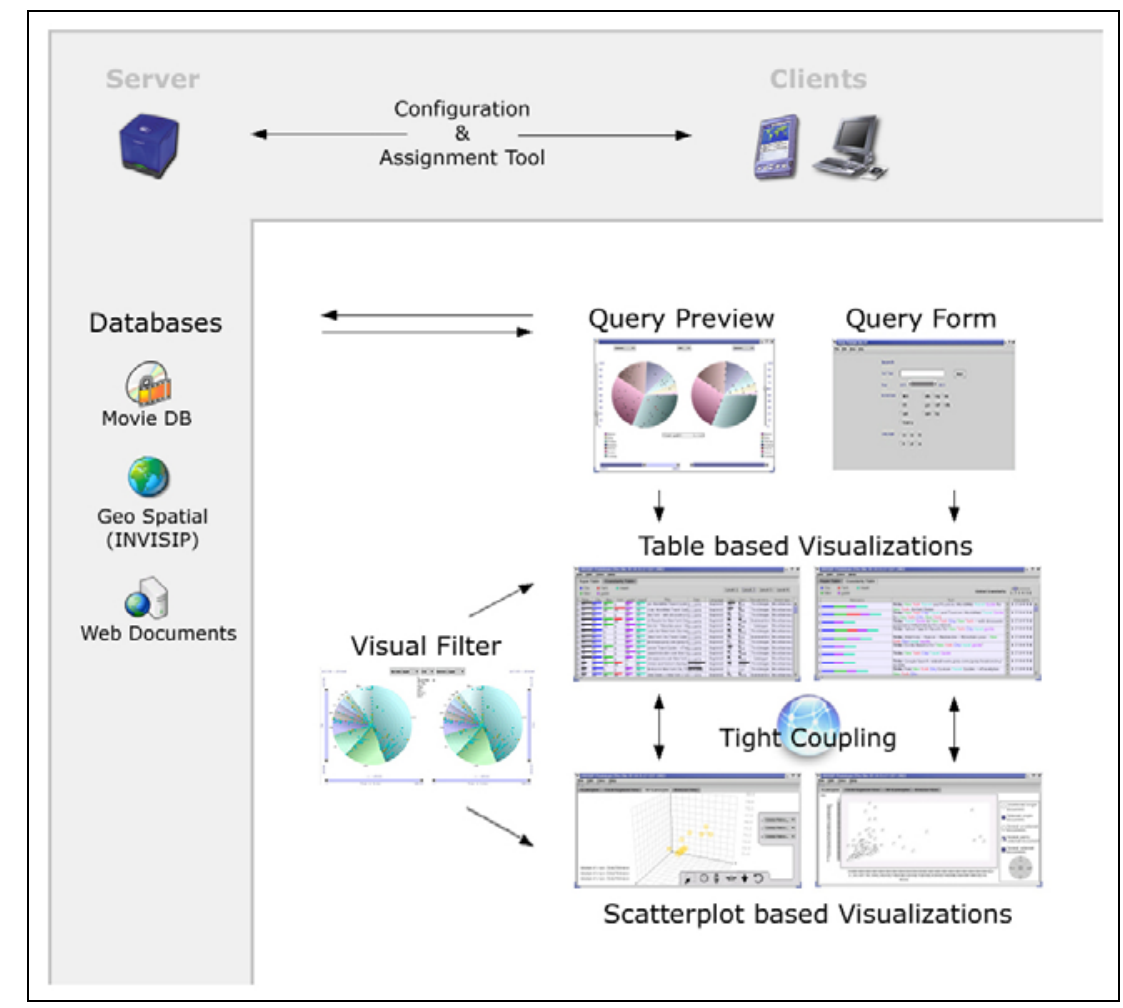

Abbildung 1: Architektur von VisMeB

Abbildung 2 zeigt eine typische Ergebnismenge in der ersten Darstellungsebene der GranularityTable. Sie ist nach Relevanz absteigend sortiert, wobei die Relevanz aus den eingegebenen Suchtermen mittels tf*idf gebildet wird. Alle anderen Metadaten sind noch nicht zu sehen, der Fokus liegt auf dem Gesamteindruck der Suche. Die Achsen des Scatterplot sind mit der Bewertung (Rank) und dem Erscheinungsjahr (Year) vorbelegt (dies kann aber vom Benutzer geändert werden). Im Scatterplot kann der Benutzer jetzt eine oder mehrere Movable Filter aufrufen, konfigurieren und mit boolschen Ausdrücken kombinieren. Movable Filter sind halbtransparente, visuelle Filter, welche alle Dokumente herausfiltern, die nicht den vom Benutzer festgelegten Kriterien entsprechen. Dokumente, welche die Filterkriterien erfüllen, nehmen in der GranularityTable (ebenso in der LevelTable) die Farbe der Movable Filter an. In Abbildung 2 hat der Benutzer zwei Movable Filter konfiguriert, der eine zeigt nur Filme mit einer Relevanz zwischen 65 und 77 (auf einer Skala von 0 bis 100), der andere nur Filme in Französisch. Beide sind kombiniert mit 
„AND“, die Schnittmenge von beiden zeigt also nur Filme an, welche beiden Kriterien genügen. Über ein Kontextmenü kann der Benutzer nun die Schnittmenge auswählen und mittels Veränderung der Granularität der Informationsdarstellung (Schieberegler im Kontextmenü mitte unten in Abbildung 2) mehr Details der ausgewählten Filme anzeigen lassen.

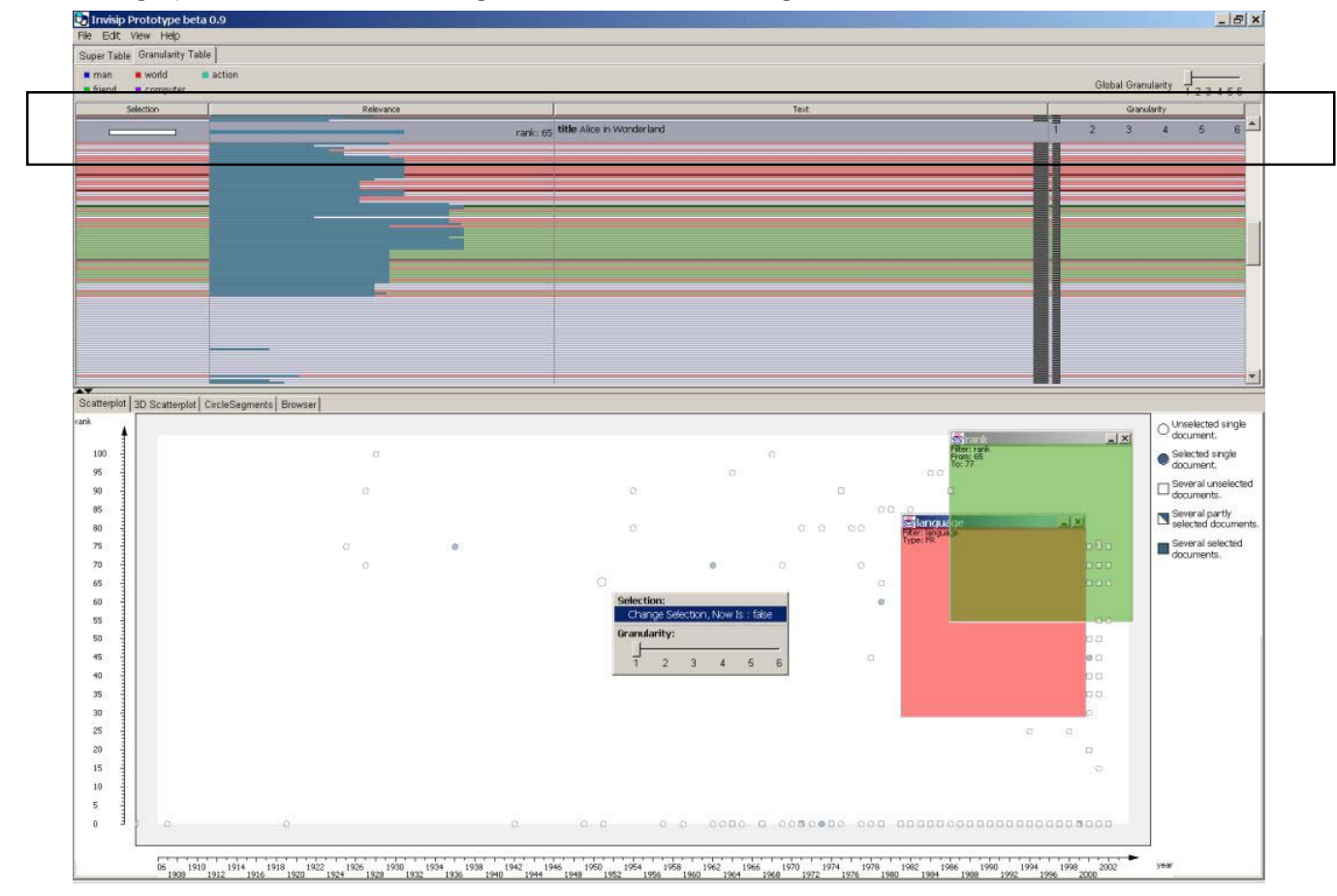

Abbildung 2: GranularityTable mit Scatterplot und Movable Filter

Prinzipiell kann er bis zur höchsten Granularität so weiter verfahren oder auch in der GranularityTable selbst über einen Schieberegler der einzelnen Dokumente (Spalte „Granularity“) mehr oder weniger über den jeweiligen Film erfahren. Dies ist besonders dann komfortabel, wenn die Treffermenge reduziert ist und zwischen den Treffern verglichen werden soll, wie in Abbildung 3 dargestellt. Die Treffer der schon reduzierten Treffermenge werden hier in unterschiedlichen Detailstufen dargestellt, ein Wechseln zwischen den Stufen ist sehr leicht möglich.

Für die Darstellung der LevelTable nutzen wir ein anderes Einsatzszenario, und zwar die Suche im Web. Dort kann die Treffermenge ungleich höher ausfallen, welches eine andere Vorgehensweise bezüglich der Reduktion der Treffermenge plausibel erscheinen lässt. Abbildung 4 zeigt eine Ergebnismenge einer Web Suchanfrage, mit den Suchtermen „New - York - City - Travel Guide“. Die erste Ebene visualisiert als tabellarische Anordnung von BarCharts einen Überblick über die Relevanzen der einzelnen Dokumente. Neben den Gesamtrelevanzen der gefundenen Dokumente werden aber auch noch die Einzelrelevanzen der Suchterme dargestellt. Um eine Vorschau auf weitere Daten zum jeweiligen Dokument zu erhalten, wurde ein Linseneffekt - basierend auf Ideen der Table Lens (siehe Rao und Card 1994) - implementiert, der eine Vorschau auf Ebene 2 ermöglicht (siehe Abbildung 4 oben). Diese Funktionalität steht auch bei der oben beschriebenen Granularity Table zur Verfügung (siehe Abbildung 2 oben), und kann weiterhin bei 
beiden Design Varianten durch den Scatterplot hervorgerufen werden. Dadurch entsteht eine enge Bindung zwischen SuperTable und Scatterplot (siehe Abbildung 4, Pfeil in beide Richtungen).

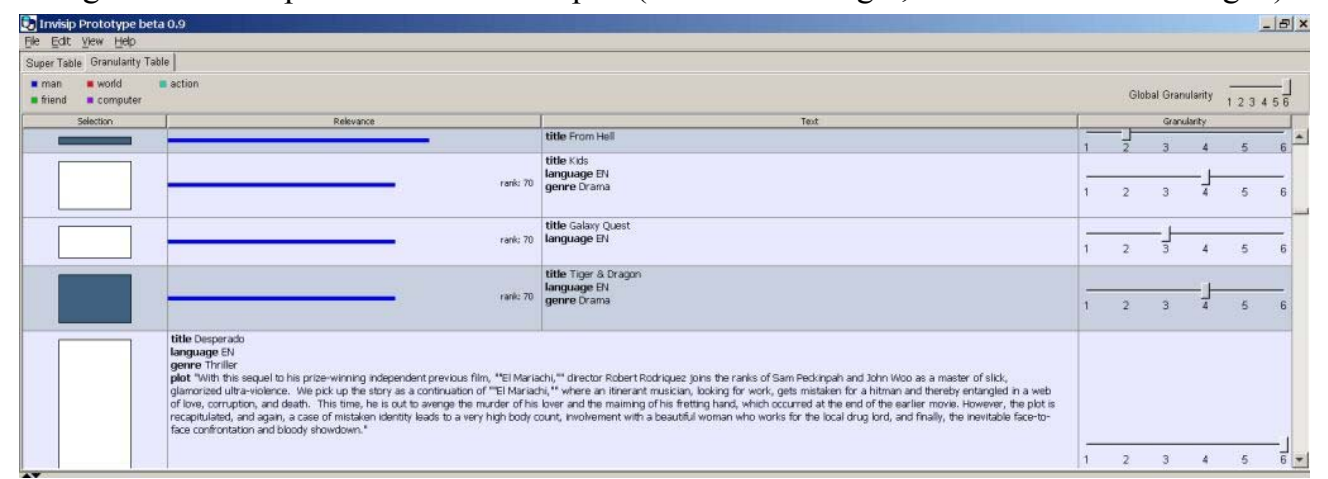

Abbildung 3: GranularityTable in unterschiedlichen Detaillierungsgraden

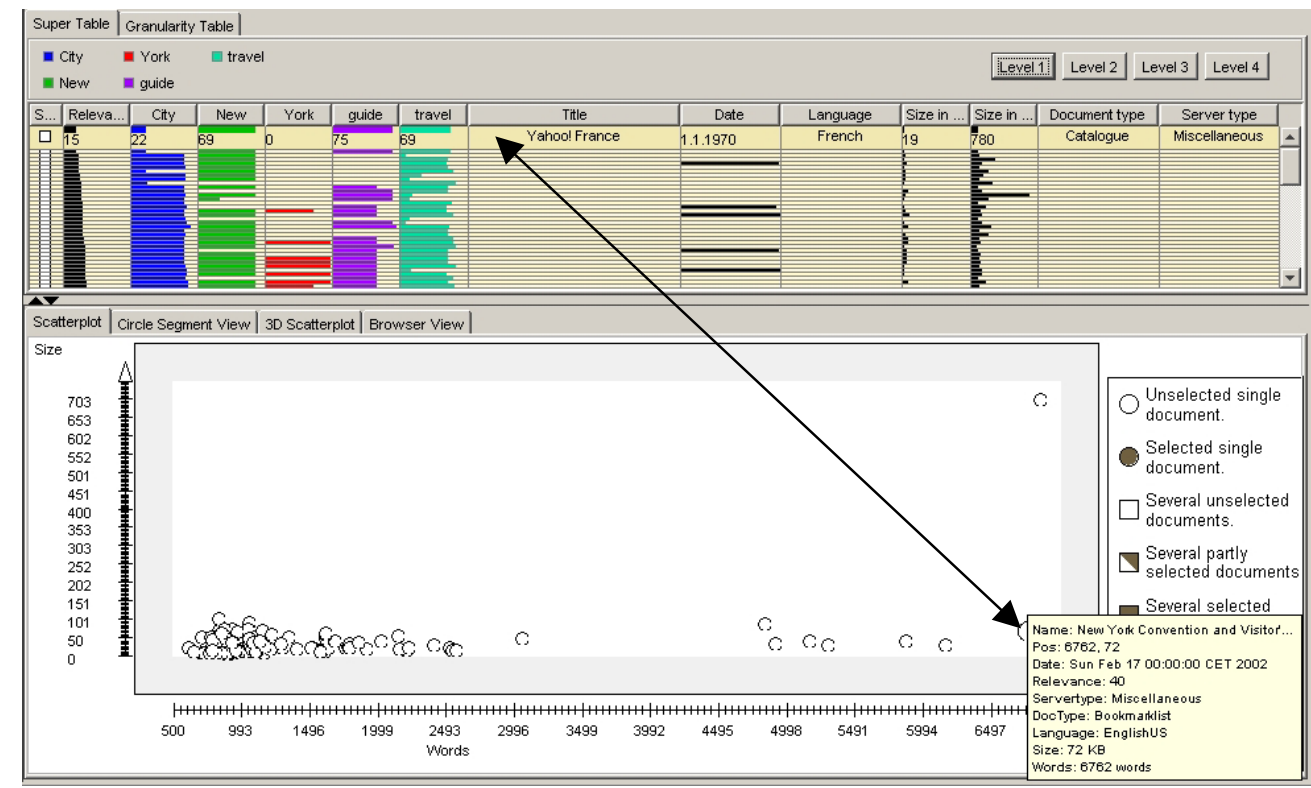

Abbildung 4: LevelTable plus Scatterplot in Ebene 1

Eine Besonderheit der LevelTable ist die Einbettung einer Relevance Curve und die Kombination mit einer Browser View. Die Relevance Curve visualisiert die Verteilung der Relevanzen der einzelnen Suchbegriffe bezogen auf einzelne Textsegmente, die Länge der Relevance Curve ist proportional zur Länge des Textes (siehe Abbildung 5). Durch eine Hervorhebung der Suchbegriffe ist eine intuitive und sehr schnelle Art der Navigation in unter Umständen langen Texten möglich. Abbildung 5 zeigt die LevelTable in Level 4 mit Relevance Curves der Dokumente, wobei der Fokus auf dem eingerahmten Dokument liegt. Hier wird per Voreinstellung der Scatterplot durch die Browser View ersetzt, d.h. der Originaltext des gerade fokussierten Dokumentes wird angezeigt. 


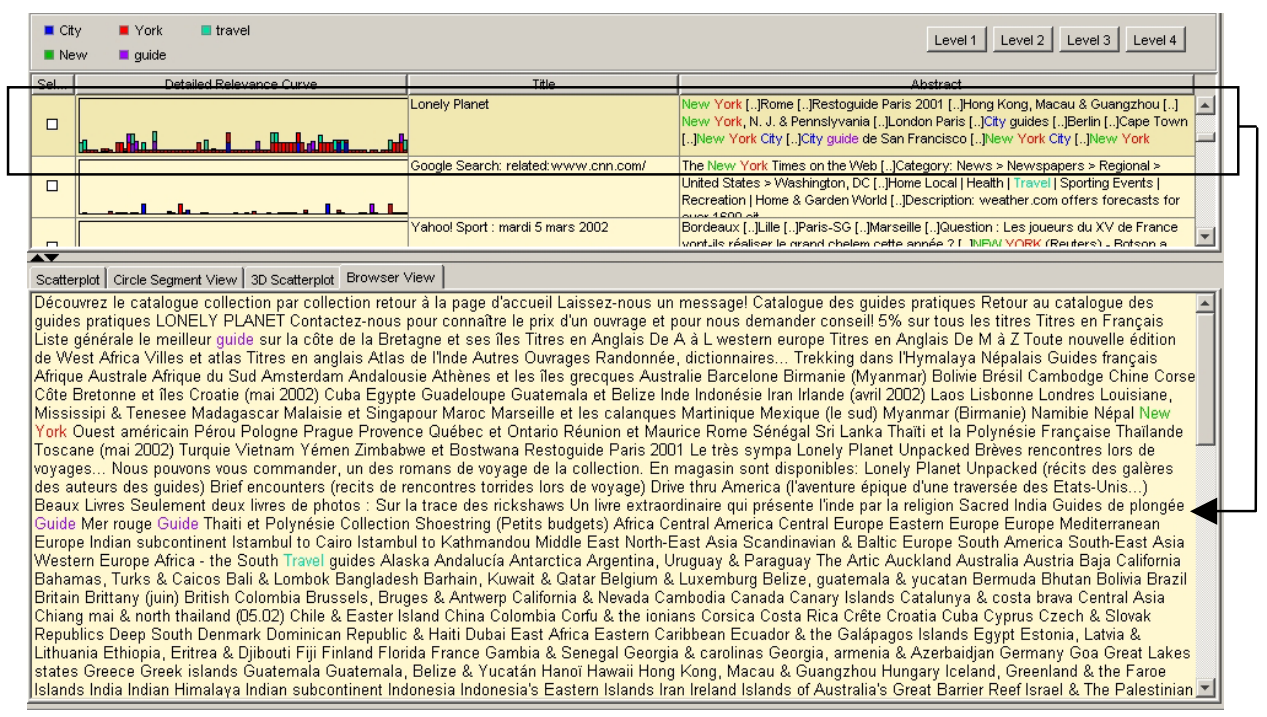

Abbildung 5: LevelTable in Level 4 mit Relevance Curve und Browser View

\section{Evaluationen}

Bei der Entwicklung von VisMeB wurden zuerst Mockups von allen wesentlichen Designvarianten entwickelt. Es handelte sich dabei um HTML-basierte Wegwerfprototypen, die einen Walkthrough anhand entwickelter Einsatzszenarien (dem scenario-based Usability Engineering von Rosson und Carroll 2002 folgend) ermöglichten. Die damit im Oktober 2002 durchgeführten Evaluationen (Benutzertests mit 8 Benutzern im eigenen Usability Lab, Probanden waren Raumplaner bzw. Projektpartner des Forschungsprojektes INVISIP) haben die prinzipielle Machbarkeit und Nützlichkeit unserer Visualisierungsideen für einen visuellen Metadatenbrowser zur explorativen Erkundung großer Datenbestände gezeigt. Evaluiert wurden die Interaktionsmöglichkeiten und das Verständnis für die verschiedenen Visualisierungen. Anhand einer simulierten Suchanfrage konnten mit den Benutzern verschiedene Schritte des Informationsgewinnungsprozesses durchgegangen werden. Durch diesen ersten Benutzertest konnten eine Reihe von Verbesserungsmöglichkeiten aufgezeigt werden, die im weiteren Verlauf des Projektes VisMeB umgesetzt wurden. So zeigte sich beispielsweise, dass das „Overview + Detail“-Konzept durch die GranularityTable besser abgebildet wird als durch den LevelTable. Dadurch kann diese Funktionalität im LevelTable in den Hintergrund treten und andere Eigenschaften dieser Visualisierung stärker betont werden. Andererseits kann die GranularityTable kaum ohne eine Einarbeitungsphase benutzt werden, wie sich in den Benutzertests gezeigt hat. Ist eine Einarbeitung einmal erfolgt, überwiegen die Vorteile gegenüber den LevelTable aber hinsichtlich der Möglichkeit, einzelne Suchergebnisse in unterschiedlichen Detaillierungsstufen miteinander parafleleichen. im den im Oktober 2002 durchgeführten Benutzertest wurde eine 6-wöchige Web-basierte Umfrage mit Hilfe eines von der AG MCI entwickelten Fragebogens gestartet. Zielgruppe waren Benutzer aus dem Bereich Raumplanung (Verwaltung und Ingenieurbüros). Insgesamt konnten 31 Fragebögen in die Auswertung übernommen werden. Die Auswertung der Web-Umfrage ergab, 
dass die Interaktion mit der LevelTable verständlicher war als mit der GranularityTable. Dazu korrelierend ergab sich in den vorherigen Benutzertests ein objektiv messbar besseres Verständnis für das Level Konzept mit seinen wählbaren festen Abstufungen im Informationsgehalt, als die über Schieberegler stetig wählbare Granularität. Es ergab sich ein interessantes Bild hinsichtlich der präferierten Suchstrategie (wurde im Fragebogen erhoben) und der präferierten Design Variante: alle der fast ausschließlich analytisch arbeitenden Befragten bevorzugten das LevelTable Design. Von den fast ausschließlich browsing-orientiert arbeitenden Befragten würde nur einer die LevelTable gegenüber der Granularity Table bevorzugen. Es ist anzunehmen, dass durch Einsatz beider Tables in unterschiedlichen Phasen des Suchprozesses deutlich effizienter und benutzergerechter gearbeitet werden kann. So könnte zunächst mithilfe der LevelTable die Treffermenge auf einen sinnvollen Umfang eingegrenzt werden, um anschließend in einem zweiten Schritt die enthaltenen Dokumente mithilfe der GranularityTable auf der inhaltlichen Ebene bequemer zu explorieren und zu beurteilen, ohne jedoch dabei die Metadaten aus den Augen verlieren zu müssen.

Ein weiterer Benutzertest mit ebenfalls 8 Probanden wurde im März 2003 (Probanden waren wieder Raumplaner des Projektes INVISIP) an einer voll funktionsfähigen Implementierung von VisMeB in Java durchgeführt werden. Primäres Ziel dieses Tests war es, die beiden Designvarianten der SuperTable miteinander zu vergleichen. Unsere Hypothese lautete, basierend auf den Erfahrungen der Web-Umfrage, dass die browsing-orientierten Benutzer die GranularityTable und die analytisch vorgehenden Benutzer die LevelTable bevorzugen würden. Unsere Annahme bezog sich auf ein Ergebnis der Web-Umfrage (siehe oben) und sollte die vermutete, je nach Suchstil präferierte Design Variante überprüfen. Diese Annahme musste verworfen werden, die Ergebnisse des Pre-Tests und die Auswertung des Benutzerverhaltens ließen keine Rückschlüsse auf unsere Annahme zu. Tabelle 1 fasst einige wesentliche Erkenntnisse der Evaluation vom März 2003 zusammen.

\begin{tabular}{|l|l|}
\hline \multicolumn{1}{|c|}{ LevelTable } & \multicolumn{1}{c|}{ GranularityTable } \\
\hline $\begin{array}{l}\text { Intuitiv, da Ähnlichkeiten zu Web- } \\
\text { Suchmaschinen }\end{array}$ & $\begin{array}{l}\text { Höherer Trainingsaufwand für } \\
\text { Verständnis erforderlich }\end{array}$ \\
\hline geringere Fehlerhäufigkeit & $\begin{array}{l}\text { TileBars sind besser mit Doku- } \\
\text { mententext verknüpft als Relevan- } \\
\text { ce Curve }\end{array}$ \\
\hline $\begin{array}{l}\text { Relevance Curve einfach zu ver- } \\
\text { stehen aber schlecht mit Doku- } \\
\text { menten verknüpft }\end{array}$ & $\begin{array}{l}\text { Besser Explorationsmöglichkeiten } \\
\text { aufgrund unterschiedlicher Detail- } \\
\text { lierungsstufen der Dokumente }\end{array}$ \\
\hline $\begin{array}{l}\text { 66\% bevorzugten Darstellung für } \\
\text { Vergleich einzelner Dokumente } \\
\text { und 80\% bevorzugten Darstellung } \\
\text { für Vergleich einzelner Suchwörter }\end{array}$ & \\
\hline
\end{tabular}

Tabelle 1: Vor- und Nachteile LevelTable/GranularityTable

Insgesamt betrachtet scheinen die Vorteile der LevelTable klar zu überwiegen. Die Benutzbarkeit dieser Designvariante wurde gegenüber der GranularityTable als durchgehend höher eingestuft. Dennoch kann letztere in besonderen Situationen (z.B. beim Vergleich kurzer aussagekräftiger Texte wie Definitionen, Lexikaeinträge etc.), in denen das „Focus \& Context“- Konzept besonders gefragt ist, von Wichtigkeit sein. Dieser Aspekt wurde von drei Probanden besonders hervorgehoben.

Es stellt sich also als Konsequenz aus den Ergebnissen der bisherigen Benutzertests die Frage, ob Level- und GranularityTable noch zukünftig gleichberechtigt nebeneinander in VisMeB einge- 
bunden werden sollen, oder ob die LevelTable primär angeboten wird, mit der Option, bei Bedarf (in Abhängigkeit von der Benutzerpräferenz) auf die GranularityTable umzuschalten. Dies soll in nachfolgenden Benutzertests mit einer größeren Anzahl von Probanden erneut untersucht werden.

\section{$5 \quad$ Resümee und Ausblick}

Die bisher entwickelten Designstudien und durchgeführten Evaluationen haben uns gezeigt, dass ein visueller Metadatenbrowser ein geeignetes Instrument zur explorativen Erkundung großer Datenmengen ist. Die bisher bei der Realisierung von visuellen Suchsystemen für bestimmte Anwendungsdomänen (Suche in einer Filmdatenbank, im Web oder in einem Metadateninformationssystem für Geodaten) gewonnenen Erkenntnisse sollen nun weiter verallgemeinert werden. Dadurch soll ein generisches Framework eines visuellen Metadatenbrowsers entstehen, das an unterschiedliche Anwendungsbereiche (mit unterschiedlichen Metadatenstandards) mit unterschiedlichen Retrievaltechniken angepasst werden kann. Dieser generische Metadaten-Browser soll zukünftig auch auf unterschiedlichen Endgeräten verfügbar sein (z. B. Desktop PCs, Tablet PCs oder Laptops, PDAs), wobei es eine noch offene Forschungsfrage ist, wie gut diese Visualisierungen bei derart unterschiedlichen Darstellungsformaten skalieren. Neben den traditionellen Interaktionstechniken (z. B. Direkte Manipulation) sollen auch multimodale Interaktionstechniken zum Einsatz kommen (z. B. Schrift- und Zeichenerkennung, Sprachsteuerung, berührungsempfindliches Display).

\section{Literatur}

Ahlberg, C.; Shneiderman, B (1994): Visual Information Seeking: Tight Coupling of Dynamic Query Filters with Starfield Displays. In: Adelson, B.; Dumais, S.; Olson, J. S. (Eds.): CHI 1994: Conference Proceedings Human Factors in Computing Systems. Conference: Boston, MA, April 24-28 1994. New York (ACM Press) 1994. p. 313-317.

Eibl, M.(2002): DEViD: a media design and software ergonomics integrating visualization for document retrieval, in: Information Visualization (2002) 1, pp.139-157

EN ISO 9241 - 11: Ergonomische Anforderungen für Bürotätigkeit mit Bildschirmgeräten - Teil 11: Anforderungen an die Gebrauchstauglichkeit - Leitsätze

Fishkin, K.; Stone, M..C.(1995): Enhanced Dynamic Queries via Movable Filters. In: Katz, Irvin R.; Mack, Robert L.; Marks, Linn et al. (Eds.): CHI 1995: Conference Proceedings Human Factors in Computing Systems. Conference: Denver, CO, May 7-11 1995. New York (ACM Press) 1995. p. 23-29.

Göbel, S.; Haist, J.; Reiterer, H.; Müller, F.(2002): INVISIP: Usage of Information Visualization Techniques to Access Geospatial Data Archives, in: Hameurlain A., Cicchetti R., Traunmüller R. (Eds.): Database and Expert Systems Applications, $13^{\text {th }}$ International Conference, DEXA 2002, Springer, pp.371-380

Hearst, M. A.(1995): TileBars: Visualization of Term Distribution Information in Full Text Information Access. In: Katz, Irvin R.; Mack, Robert L.; Marks, Linn et al. (Eds.): 
CHI 1995: Conference Proceedings Human Factors in Computing Systems. Conference: Denver, CO, May 7-11 1995. New York (ACM Press) 1995. pp. 5966.

Klein, P.; Müller, F.; Reiterer, H.; Eibl, M.(2002): Visual Information Retrieval with the SuperTable + Scatterplot, in: Proceedings of the 6th International Conference on Information Visualisation (IV 02), IEEE Computer Society, 2002, S.70-75

Mann, T. M. (2002): Visualization of Search Results from the World Wide Web, Dissertation, University of Konstanz, 2002, http://www.ub.uni-konstanz.de/kops/volltexte/2002/751/

North, C. L.; Shneiderman, B.(2000): Snap-Together Visualizations: Can Users Construct and Operate Coordinated Views. In: International Journal of Human-Computer Studies, 53 (2000) 5, p. 715-739.

Nowell, L. T.; France, R. K.; Hix, D. et al.: Visualizing Search Results: Some Alternatives to Query-Document Similarity. In: Frei, Hans-Peter; Harman, Donna K.; Schäuble, Peter et al. (Eds.): SIGIR 1996: Proceedings of the 19th Annual International ACM SIGIR Conference on Research and Development in Information Retrieval. Conference: Zürich, Switzerland, August 18 -22 1996. New York (ACM Press) 1996. p. 67-75.

Rao, R.; Card, S. K.(1994): The Table Lens. Merging graphical and symbolic representations in an interactive focus + context visualization for tabular information. In: Adelson, B.; Dumais, S.; Olson, J. S. (Eds.): CHI 1994: Conference Proceedings Human Factors in Computing Systems. Conference: Boston, MA, April 24-28 1994. New York (ACM Press) 1994. pp. 318-322.

Reiterer, H.; Mußler, G.; Mann, T.; Handschuh, S.(2000): INSYDER - An Information Assistant for Business Intelligence, Proceedings of the 23 Annual International ACM SIGIR 2000 Conference on Research and Development in Information Retrieval, ACM press, 2000, pp.112-119

Reiterer, H.; Mußler, G..; Mann, T.(2001): A visual Information seeking system for Web search, in: Oberquelle H., Oppermann R., Krause J. (Hg.), Mensch \& Computer 2001, Teubner, Stuttgart, 2001, S.297-306

Rosson, M.; Carroll, J.(2002): Usability Engineering - Scenario-based Development of HumanComputer Interaction, Morgan Kaufmann, 2002

Spence, R.; Apperley, M.D.(1982): Data Base Navigation: An office environment for the professional, in: Behaviour and Information Technology, 1982, 1, 1, pp. 43-54

Spenke, M.; Beilken, C.; Berlage, T. (1996): FOCUS: The Interactive Table for Product Comparison and Selection. In: UIST 96: 9th ACM Symposium on User Interface Software and Technology New York (ACM Press) 1996. p. 41-50.

Tanin, E.; Plaisant, C.; and Shneiderman, B.(2000): Browsing Large Online Data with Query Previews, in: Proceedings of the Symposium on New Paradigms in Information Visualization and Manipulation - ACM CIKM, pp. 80-85, 2000, USA

Veerasamy, A.; Navathe, S. B(1995): Querying, Navigating and Visualizing a Digital Library Catalog. In: Digital Libraries 1995: The Second Annual Conference on the Theory and Practice of Digital Libraries. Conference: Austin, TX, June 11-13 1995. http://www.csdl.tamu.edu/DL95/papers/veerasamy/veerasamy.html [1999-0324] 


\section{Adressen der Autoren}

Prof. Dr. Harald Reiterer / Tobias Limbach / Frank Müller / Peter Klein / Christian Jetter Universität Konstanz

FB Informatik und Informationswissenschaft

Postfach D73

78457 Konstanz

harald.reiterer@uni-konstanz.de

tobias.limbach@uni-konstanz.de

frank.mueller@uni-konstanz.de

peter.klein@uni-konstanz.de 